\title{
Two Formal Interpretations of Bolzano's Theory of Substances and Adherences
}

\section{Kordula Świętorzecka ${ }^{1}$}

Received: 21 December 2017 / Accepted: 20 May 2018 / Published online: 27 July 2018

(c) The Author(s) 2018

\begin{abstract}
Our research concerns a formal representation of Bolzano's original concepts of Substanz and Adhärenz. The formalized intensional theory enables to articulate a question about the consistency of a part of Bolzano's metaphysics and to suggest an answer to it in terms of contemporary model theory. The formalism is built as an extension of Zalta's theory of abstract objects, describing two types of predication, viz. attribution and representation. Bolzano was aware about this distinction. We focus on the consistency of this formalism and the description of its semantics. Firstly, we explore the possibility to reconstruct a Russellian antinomy based on the concept of the Bolzano's Inbegriff of all adherences. (Bolzano's theory of ideas is often suspected of antinomial consequences.) Our aim is to show limitations of his theory that prevent a contradiction when the Inbegriff consists of non-selfreferential adherences. Next, we discuss two competing semantics for the proposed theory: Scott's and Aczel's semantics. The first one yields a problematic result, that there are no models for the considered theory, containing a non-empty collection of all adherences. This is due to the fact that Scott's structures verify the formula on reloading abstracts in extensional contexts. We show that Aczel's semantics does not contain this difficulty. There are described Aczel's models with a non-empty set of all adherences. The self-referentiality of such a collection becomes irrelevant here. Finally, we show that there are Aczel's structures verifying the formula on reloading abstracts and we exclude them from the class of models intended for our theory.
\end{abstract}

Keywords Formal ontology · Intensional logic · Bolzano's metaphysics · Substance · Adherence $\cdot$ Abstract objects

The metaphysics of Substanz and Adhärenz is a part of Bolzano's philosophy, which may be of interest both for historical and systematic reasons. It is characterized by Aristotelian categories with Platonic (Leibnizian) meanings combined in an original

Kordula Świętorzecka

k.swietorzecka@uksw.edu.pl

1 Cardinal Stefan Wyszyński University in Warsaw, Warsaw, Poland 
way, a metaphysical approach that stands behind many of Bolzano's philosophical views. At the same time, Bolzano's theory of substances and adherences together with parts of his broader ontology constitute an original philosophical framework, distinct from its historical relative-the Meinongian theory of objects.

Our research is of systematic character and it concerns a formal representation of Bolzano's original concepts of substance and adherence. We consider a formalized intensional theory, which enables us to precisely ask a question about the consistency of Bolzano's ideas and to suggest an answer to it in terms of contemporary model theory.

The starting point of our analysis is a formalization introduced by Świętorzecka (2017) to reconstruct Bolzano's argument for the existence of substances formulated in Athanasia (Bolzano 1827). The argument is based on the concept of the Inbegriff of all adherences - a collection of properties (Beschaffenheiten) of a certain kind that occur in (befindet sich an) substances or in other adherences. Bolzano claimed that the existential dependence of adherences on their subjects is inherited by the resulting collection (Inbegriff) of properties, which in turn led him to conclude that at least one individual exists, namely a substance. The formalization that we will consider assumes that an adequate description of Bolzano's intentions should employ two types of predication. Indeed, the distinction between the contexts in which adherences are treated as attributes of individuals and the contexts in which they are considered as individuals gathered together (in one Inbegriff), enables one to note an important intensional aspect of the formalized reasoning. In turn, that may help one to find further connections between the Bolzanian theory of adherences and the part of his theory of objects that deals with so called ideas in themselves (Vorstellungen an sich). These two types of predication, viz. attribution (to individuals) and representation (of properties) are described in Zalta's theory of abstract objects (Zalta 1983), which is the formal background of our proposal. We offer new interpretative details and introduce a change in the axiomatics given by Świętorzecka (2017) to get the derivation of the thesis about the existence of a substance. We contend that this strategy is closer to Bolzano's original idea.

The main focus of our approach is on the consistency of our formalism and the description of its semantics. Firstly, we explore the possibility to reconstruct a Russellian antinomy based on the concept of the Inbegriff of all adherences. It is well known that Bolzano's theory of ideas is suspected of antinomial consequences, especially when general concepts such as the idea of an object or the idea of something in general are considered. Our aim is to show certain limitations of Bolzano's theory that prevent a contradiction when the Inbegriff consists of non-self-referential adherences. Secondly, we discuss two competing semantics for our formalization. The first one-Scott's semantics-was originally designed for Zalta's theory of abstract objects. Although it verifies all axioms of our theory, it also assigns to it 'trivial' models in which the truth of certain specific implicational axioms is guaranteed by the falsehood of their antecedents, which assume the existence of an Inbegriff of all adherences. As we will see, Scott's semantics yields an even more problematic result, namely, that there are no Scott models for the considered theory, containing a non-empty collection of all adherences. This is due to the fact that Scott's structures verify the formula on reloading abstracts in extensional contexts which means that 
if any abstract individual belongs to the extension of any property, then all abstracts belong to it. This observation alone gives enough motivation to investigate another possible semantical framework for our theory. To this end, we turn to the semantics elaborated by Zalta (1997, 1999), which was inspired by Aczel. We adopt a version of Aczel's structures and show models with a non-empty set of all adherences. We argue that the question of the self-referentiality of such a collection becomes irrelevant here. Finally, we show that there are Aczel's structures verifying the formula on reloading abstracts and we exclude them from the class of models intended for our theory.

\section{Philosophical Background and Formal Framework}

In this section we briefly present the main ideas formulated by Świętorzecka (2017), but adding a number of changes to them. We also revise some translations of the original texts and refine their interpretation in light of additional sources. Most of these modifications are inspired by suggestions from Professor Wolfgang Künne.

\subsection{Bolzano on Substance-Adherence, and the Collection of All Adherences}

In Bolzano's philosophical system, the concepts of substance and adherence function as metaphysical categories (WL, I.560). ${ }^{1}$ The first extensive passage in which this pair occurs comes from Athanasia - the work in which Bolzano tries to defend the hypothesis of the immortality of the human soul. There, Bolzano sketched an argument for the existence of substances based on the concept of adherence. ${ }^{2}$ This argument is reconstructed in our formalization:

[1] If there is one actual [Wirkliches] thing, it is evident that there must be one or more than one substances. [2] For an actual thing that is not a substance must be an adherence, hence a property [Beschaffenheit], and so it presupposes another actual thing in which it occurs [befindet sich an]. [3] This other actual thing might in turn be an adherence or property because properties, too, have their own properties, and if the former are actual things then so are the latter. [4] But, if we think of all actual things that are merely adherences as united in one collection [Inbegriff], it soon becomes clear that this [collection], too, cannot exist [bestehen] by itself, but it requires one or several other actual things in which it occurs. [5] Now this one or these several actual things are not again adherences, for if they were they would belong to this collection; so they are

\footnotetext{
1 References are to volume number and page number of the original German edition of WL (Bolzano 1837a). Readers may find detailed references to Bolzano's texts and an extensive study of these concepts especially by Künne (2015) and Schnieder (2002). We refer only to the passages that are relevant to our later approach introduced in Sect. 2.

2 Bolzano distinguished between simple substances and complex substances (aggregates of the former), and argued for the existence of simple substances from the existence of such aggregates (cf. Bolzano $1851, \S 50-65)$.
} 
substances. [6] Therefore, it is certain that there are substances as it is certain that there is at least one actual thing.

(original text: Bolzano, ed. 1838, 39, we take author's translation, cf. Świętorzecka 2017)

The adjective 'wirklich' is translated as 'actual' (from 'wirken'- 'to act'). Throughout the whole text we adopt Künne's translation for most key Bolzanian terms (Künne 2008).

The above argument directly applies to souls only if Bolzano understands 'substance' as 'simple substance'. Bolzanian substances, if there are any, are actual things, that is, they are elements of the causal order. They are bearers of adherences, which also are actual things. Such substances are simple, they are not perceivable, and exist omnitemporally (human souls are supposed to be a subclass of them). In many respects Bolzanian simple substances are like Leibnizian monads, but interact with each other. ${ }^{3}$ Substances and adherences constitute the realm of all actual things. Bolzano's general notion of a substance and his distinction between substances and adherences is clearly inspired by Aristotle's dichotomy of substances and accidents. Aristotelian substances possess their accidents as attributes (cf. Aristotle $1928 \mathrm{Met}$ 1028a-29; Cat 15b; Met 1022b). The same applies to Bolzano's substances and adherences (WL, I.379), although for Bolzano the relation of possessing may also occur between adherences, when one adherence is the subject of another (cf. [3] in the block quotation above). In order to grasp Bolzano's concept of adherence, let us focus on a few examples from Athanasia. There, he claims that the color, smell, and weight of a given body are adherences $(1838, \S 1)$. An adherence is also this redness of a given flower $(\$ 1,24)$. Unusual power may also be an adherence of memory, which in turn is an adherence of any given person $(\S 1,23) .{ }^{4}$

Possession of an attribute by a subject is expressible in Bolzano's system by a sentence in its canonical form: $A$ has $b$, where " $b$ represents the abstractum that belongs to the concretum $B$ " as used in the traditional construction: $A$ is $B$ (WL, II.9). Bolzano's canonical sentences consist of two terms connected by the copula has. ${ }^{5}$ Sentences express (drücken aus) the so-called propositions in themselves (Sätze an sich), which are built from ideas in themselves represented by the terms $A, b$ and the copula has. Propositions and ideas in themselves form the Bolzanian world of non-actual objects.

The canonical form $A$ has $b$ may be substituted with a certain restriction:

\footnotetext{
3 Bolzano's diary notes entitled Verschiedenheit zwischen Leibnitzens und meinem Ansichten (Bolzano 1837b) are very informative for this comparison (they were overlooked by Świętorzecka (2017)).

${ }^{4}$ Examples such as these seem to show that possessing is neither reflexive, nor symmetric, or transitive. These features are not captured by our formalization. We shall also put aside the fact that Bolzano distinguishes between properties of actual objects that are identity-dependent on their bearers and properties of actual objects that are shared by several objects. The former are particulars, that latter universals.

${ }^{5} \mathrm{He}$ gives many examples of reduction of sentences $A$ is $B$ to sentences $A$ has $B$. There are considered, among others, also reductions of existential and normative sentences: $A$ is (exists) is equivalent with A-has-existence; A should do (be) ...- A-has-an obligation to do (be) ... (WL, II.10-15).
} 
If the opposition between substance and adherence is to be exactly grasped and preserved then one has to understand by substance only that actual thing (an entity) that can only be thought of as a subject, never as a predicate.

(Bolzano 1833, 144)

Thus, the variable $b$ in the scheme $A$ has $b$ cannot represent any substance. The variable $A$, however, may be substituted by terms designating (bezeichnen) substances or adherences.

According to Bolzano, the term $b$ in $A$ has $b$ designates an adherence if, and only if $A$ designates an actual object, and $A$ has $b$ expresses a truth. If these conditions are fulfilled, we can call $b$ an 'adherential term' and the idea expressed by it an 'adherential idea'. Thus understood, an adherential idea is never empty or, as Bolzano would put it, objectless (gegenstandlos).

The one-to-one relationship between adherential terms and adherential ideas will be used in our rather simple interpretation of Bolzanian Inbegriff of all adherences. Let us focus one of the many meanings of the term 'Inbegriff', explained in WL (I.393) as a collection of individually listed objects:

If $[\ldots]$ we are supposed to indicate the cause of a certain result $M$, and it turns out that several objects $A, B, C, D, \ldots$ contributed to its production, ${ }^{6}$ we must, in order to think of the complete cause of $M$, think of $A, B, C, D, \ldots$ together, that is, think of the collection of $A, B, C, D, \ldots$ And it is obvious, that [...] we do not think about the order and sequence in which they contributed to bringing about $M$.

(WL, I.394)

Surely, adherences are not the cause of substances. However, all of them taken together need (require, bedürfen) existence of substances. We consider the components of such a whole in an intensional way. The very concept of Inbegriff of all adherences will get both extensional and intensional interpretation. We take as the extensional counterpart of this concept, a set of all adherential ideas. From the other hand, it will be also represented by certain intensional object attributed to every adherential idea on the basis of inherence relation. What we should say is, that the proposed extensional interpretation may be considered as not adequate in case of Bolzanian Inbegriff in general. ${ }^{7}$ However, our approach is just not general but only dedicated to adherential Inbegriff. It enables us to trace the original structure of the analyzed argument and for this reason, we think that our solution has explanatory power.

\footnotetext{
${ }^{6}$ Later in the text Bolzano adds "no matter how great the number of $A, B, C, D, \ldots$ may be" (ibid., I.395).

${ }^{7}$ Let us note, that there is also a mereological interpretation of Bolzano's Inbegriff of all conditioned beings in a reconstruction of one of his arguments for the existence of God (cf. Świętorzecka 2014). The problem of general interpretation of Inbegriff is considered by Lapointe (2011, 116-197). As it is claimed "Bolzano's collections (Inbegriffe) are neither sets, nor mereological sums, nor classes" (116).
} 


\subsection{Zalta's AOT Theory}

The framework we will consider is a fragment of a rich formal ontology originally proposed by Zalta (1983). The first attractive feature of this formalism is the structure of its language. Zalta uses predicate terms that represent the so-called gerundive versions of certain verbs, predicate adjectives, and predicate nouns (Zalta 1983, 37-38). The predication analysed by Bolzano assumes the same grammar when he tries to bring all simple sentences into the canonical form $A$ has $b$. Secondly, Zalta's language enables one to distinguish between extensional and intensional contexts. To this end two different concatenations of individual and predicate terms are used. Formulas $\tau x$ read as follows: (individual) $x$ exemplifies (the property) $\tau$ and mean that some individual object belongs to the extension of a certain property. Formulas $x \tau$-(individual) $x$ (represents) encodes (the property) $\tau$ are used to describe the fact that some individual belongs to the intension of a certain property. Bolzano was obviously aware of the extension-intension distinction. In his account, ideas in themselves that are not objectless may have the same extension (Umfang) even though they are different ideas. Also, an idea in itself is an intensional object that can be both the Stoff (matter, content) of thinking and the Sinn (sense) of a linguistic expression used to express the act of thinking (Künne 1997). Zalta's theory deals specifically with Meinongian abstract objects, which have the status of individuals. We claim that simple Bolzanian ideas have exactly the same status, although their number and variety are much more modest than Meinongian objects. This difference is kept in our proposed formalization of Bolzano's concepts. We contend that the specific axiom schema assumed by Zalta to introduce 'the paradise' of Meinong's abstracts, is not used in its full range.

We start our brief presentation with a description of the language employed. We use $x$ as a symbol for individual variables: $x, y, z, \ldots ; F$ for predicate variables: $F, G, \ldots$; the primitive constant $E$ ! reads as follows: exists (concretely); the binary constant $=_{E}-i$ (concretely) identical with; the logical symbols: $\neg, \rightarrow, \forall, \lambda$ and parentheses.

The predicate terms and formulas are the following:

$$
\begin{aligned}
& \tau::=F|E !| \lambda x \varphi \\
& \varphi::=\tau x|x \tau| x=_{E} x|\forall x \varphi| \forall F \varphi|\neg \varphi| \varphi \rightarrow \varphi
\end{aligned}
$$

Expressions having the form $x \tau$ are called encoding formulas. Propositional formulas are expressions that do not contain encoding subformulas nor quantifiers for predicate variables.

Let us use $\alpha, \beta$ as representing individual or predicate variables. Symbol t represents individual or predicate terms.

The assumed logic is characterized by classical propositional tautologies and all instantiations of schemata:

( $\forall 1) \forall \alpha \varphi \rightarrow \varphi_{\alpha}^{\mathrm{t}}$, t is substitutable for $\alpha$

$(\forall 2) \forall \alpha(\varphi \rightarrow \psi) \rightarrow(\varphi \rightarrow \forall \alpha \psi), \alpha$ is not free in $\varphi$

$(\operatorname{Comp} \lambda)[\lambda x . \varphi] y \leftrightarrow \varphi_{x}^{y}$, where $\varphi$ is a propositional formula. 
Primitive rules are: (modus ponens) $\vdash \varphi \rightarrow \psi, \vdash \varphi \Longrightarrow \vdash \psi$ and (generalization) $\vdash \varphi \Longrightarrow \vdash \forall \alpha \varphi$.

The theory of abstract objects AOT is characterized by adding the following proper axioms and definitions:
A1 $x={ }_{E} y \leftrightarrow E ! x \wedge E ! y \wedge \forall F(F x \leftrightarrow F y)$
(identity of concretes)
A2 $E ! x \rightarrow \neg \exists F(x F)$
(concretes are no-coders)
D1 $A x=_{d f}[\lambda y \cdot \neg E ! y] x$
(abstract object)
D2 $F=G={ }_{d f} \forall x(x F \leftrightarrow x G)$
(intensional identity)
A3 $\exists x(A ! x \wedge \forall F(x F \leftrightarrow \varphi(F)))$ $x$ is not free in $\varphi$
(being-so of A!-objects)
D3 $x=y={ }_{d f} x={ }_{E} y \vee(A ! x \wedge A ! y \wedge \forall F(x F \leftrightarrow y F))$
$\mathrm{A} 4 \alpha=\beta \rightarrow\left(\varphi \alpha \leftrightarrow \varphi\left[\begin{array}{l}\beta \\ \alpha\end{array}\right]\right)$
(substantiation of identicals)

Axiom A3 is assumed by Zalta to express the so-called Meinongian principle of the independence of being from being-so. ${ }^{8}$ Abstract objects, like all objects, existso, even when they represent empty properties (like the property of being a round square). This perspective departs from Bolzano's strategy: his ideas mustn't have empty extensions, otherwise they are objectless. In the context of the above formalism, 'a surplus' of abstracts is irrelevant in our framework. We do not assume that all abstract individuals acknowledged by Zalta's theory are to be considered as Bolzanian ideas. Furthermore, we claim that abstracts, considered as ideas of adherences, are not objectless: adherences are always attributes of something.

We will use a few theses and definitions of AOT.

T1 $\quad \exists^{1} x(A ! x \wedge \forall F \neg x F)$

where $\exists^{1} \alpha \varphi$ is an abbreviation for $\exists \alpha\left(\varphi \wedge \forall \beta\left(\varphi_{\alpha}^{\beta} \rightarrow \beta=\alpha\right)\right)$

We introduce the following constant for abstract object that does not encode any property $^{9}$

\footnotetext{
${ }^{8}$ Actually, the restriction of $\operatorname{Comp} \lambda$ according to which $\varphi$ must be a propositional formula is linked with the acceptance of A3. Not restricted Comp $\lambda$ with A3 and A4 leads to a contradiction. We will come back to this point in Sect. 3 (cf. footnote 13). A brief description of alternative ways to speak consistently about Meinong's abstracts is given for instance in Rapaport (1978).

${ }^{9}$ Constant $\mathrm{o}$ is called null by Świętorzecka (2017) but perhaps this label would be more adequate for representations of properties with empty extensions.
} 
D4 $x=\mathrm{o}={ }_{d f}(A ! x \wedge \forall F \neg x F) \quad$ (invisible abstract)

We further assume that every no-coder that is visible, is a concrete:

T2 $\forall F \neg x F \wedge \neg x=0 \rightarrow E ! x$

To this, we add Zalta's definition of a characteristic coder:

D5 $x \operatorname{con} F={ }_{d f} \forall G(x G \leftrightarrow G=F)$ (x characterizes $F$ )

The following theses are provable inter alia:

T3a $x \operatorname{con} F \wedge x \operatorname{con} G \rightarrow F=G$

T3b $x \operatorname{con} F \wedge x G \rightarrow F=G$

T4a $\forall F \exists x(x \operatorname{con} F)$

T4b $\forall F \exists^{1} x(x \operatorname{con} F)$

\section{Theory $\mathrm{AOT}_{A d B \mathfrak{l}}[\mathfrak{B}]$}

In this section we form a theory motivated by Bolzano's concepts of substance and adherence.

We use $\mathrm{T}[X]$ to name a theory obtained from the theory $\mathrm{T}$ by adding a set $X$ of formulas or a single formula $X$ as axioms to it. In the lower index we note the new primitive constants added to the $T$ language.

Formulas $\tau x$ are now to be read as $x$ has (the property) $\tau$.

The language is enriched by adding two constants $A d$ and $B$, used in contexts $A d \tau, B \tau$ to be read as: (property) $\tau$ is an adherence; (property) $\tau$ is conditioned (by something actual).

Firstly, we formulate a thesis about the Bolzanian translation of sentences having the general form $A$ is $B$ into sentences having the general form $A$ has $b$.

We define the concept of inherence:

D6 $x \varepsilon y=_{d f} \exists F(F x \wedge \neg x=\circ \wedge y c o n F)$

and prove that:

TB1 $x \varepsilon y \wedge y \operatorname{conF} \rightarrow F x$

We identify Bolzanian substances with Zalta's existing individuals:

$$
\text { D7 } S x=_{d f} E ! x
$$

(substance)

The theory of abstracts objects expressed in the above language will be called $\mathrm{AOT}_{A d B}$. 
To give an explanation of Inbegriff we use the predicate term $\mathfrak{I} \mathfrak{n}$ introduced following Comp $\lambda$. However, our proposal breaks the restriction added to Comp $\lambda$. For this reason all $\mathfrak{I} \mathfrak{n}$ substitutions of logical tautologies, A3 and A4 must be weakened by the condition about the existence and uniqueness of a property named just by $\mathfrak{I}$ :

$$
\text { IN } \quad \exists^{1} Z \forall x(Z x \leftrightarrow \exists F(A d F \wedge x \operatorname{con} F))
$$

Now we list the following axioms motivated by the previously quoted passage from Athanasia and some of Bolzano's remarks on substances and adherences: ${ }^{10}$

$$
\begin{aligned}
& \text { IN } \rightarrow \forall x(\Im \mathfrak{I} x \leftrightarrow \exists F(A d F \wedge x c o n F)) \\
& \text { If the Inbegriff of all adherences is consistent, then } x \text { has it iff } x \text { is the } \\
& \text { characteristic coder of some adherence. } \\
& \text { (cf. [4] ) }
\end{aligned}
$$

$\mathrm{AB} 1 \quad \exists x S x \vee \exists F A d F$

There exists something actual: a substance or an adherence. (cf. [1], [2])

$\mathrm{AB} 2 \quad I N \rightarrow(\exists x \mathfrak{I n} x \rightarrow B \mathfrak{I n})$

If the Inbegriff of all adherences is consistent and has not empty extension, then it is conditioned by something actual.

AB3 $\quad \forall F(B F \rightarrow \forall x(F x \rightarrow S x) \vee \exists x(F x \wedge \exists y(y \varepsilon x \wedge \neg F y)))$

Every property $F$ conditioned by something actual, is a property of a substance or a property of something which is attributed to an individual which has no $F$.

AB4 IN $\rightarrow(A d F \wedge F x \rightarrow S x \vee \mathfrak{I n} x)$

If the Inbegriff of all adherences is consistent and $x$ exemplifies any adherence, then $x$ is a substance or $x$ characterizes some adherence.

Let us call the set of the above axioms $\mathfrak{B}$.

Theory $\mathrm{AOT}_{A d B \mathfrak{I n}}[\mathfrak{B}]$ is a proposed formalization of the considered part of Bolzano's lecture on substances and adherences.

Our AB4 differs from its previous version of Świętorzecka (2017). ${ }^{11}$ It is a simpler and more intuitive formulation of the assumption that justifies [5]. Let us also note that AB4 follows from $A d F \wedge F x \wedge \forall G(A d G \rightarrow \neg x c o n G) \rightarrow S x$ which could be called the principle of the substantial foundation of adherences.

We now modify the proof of the main thesis, which is a counterpart of the theorem justified by Bolzano in Athanasia, weakened by condition IN:

\footnotetext{
${ }^{10}$ We refer to numbers introduced in the quoted Bolzano's argumentation to note the inspiration for our axioms.

${ }^{11}$ Originally, A4. IN $\rightarrow(\mathfrak{I n} x \wedge \neg x \operatorname{con} S \wedge \neg \mathfrak{I n} y \wedge y \varepsilon x \rightarrow \exists z(y \varepsilon z \wedge z \operatorname{conS}))$ was called the principle of substantial foundation of $\mathfrak{\Im} \mathfrak{n}$.
} 
TB2 $I N \rightarrow \exists x S x$

1. $\exists x S x \vee \exists F A d F$

$\mathrm{AB} 1(=1 a \vee 1 b)$

1b. $\exists F A d F$

ass

2. $\exists F \exists x(A d F \wedge x \operatorname{con} F)$

T4a, $1 b$

3. $I N \rightarrow \exists x \mathfrak{I n} x$

AB0, 2

4. $I N \rightarrow B \mathfrak{I n}$

$\mathrm{AB} 2,3$

5. $\quad I N \rightarrow(B \mathfrak{I n} \rightarrow \forall x(\mathfrak{I n} x \rightarrow S x) \vee \exists x(\mathfrak{I n} x \wedge \exists y(y \varepsilon x \wedge \neg \mathfrak{I n} y)))$

AB3, $\forall 1$

6. $\quad I N \rightarrow \forall x(\mathfrak{I n} x \rightarrow S x) \vee \exists x(\mathfrak{I n} x \wedge \exists y(y \varepsilon x \wedge \neg \mathfrak{I n} y))$

4,5

6a. $\quad I N \rightarrow \forall x(\mathfrak{I n} x \rightarrow S x)$

ass

7. $\quad I N \rightarrow \forall x(\mathfrak{I n} x \rightarrow \exists F x \operatorname{con} F)$

AB0

8. $I N \rightarrow \forall x(\mathfrak{I n} x \rightarrow \exists F x F)$

D5, 7

9. $\quad I N \rightarrow \forall x(\mathfrak{I n} x \rightarrow \neg S x)$

8, A2, D7

10. $I N \rightarrow \exists x(\mathfrak{I n} x \wedge \exists y(y \varepsilon x \wedge \neg \mathfrak{I n} y))$

$6,6 a, 9,3$

11. $I N \rightarrow \exists x(\exists F(A d F \wedge x \operatorname{con} F) \wedge \exists y(\exists G(G y \wedge x \operatorname{con} G) \wedge \neg \mathfrak{I n} y))$ 10, AB0, D6

12. $I N \rightarrow \exists x(\exists F(A d F \wedge x \operatorname{con} F \wedge \exists y(F y \wedge x \operatorname{con} F \wedge \neg \mathfrak{I n} y))$

11, T3a

13. $I N \rightarrow \exists F \exists y(A d F \wedge F y \wedge \neg \mathfrak{I n} y)$

14. $I N \rightarrow \exists x S x$

$13, \mathrm{AB} 4$

\section{More About $\mathrm{AOT}_{A d B \mathfrak{l}}[\mathfrak{B}]$ : Towards a Russellian Antinomy}

Our theory states that:
TB3 $\exists^{1} x(x \operatorname{con} F \wedge F x) \rightarrow \neg B F$
(A2, AB3, T3a, D1)
A property exemplified only by its own characteristic coder is not con- ditioned.

We introduce the concept of universal property

$$
\text { D8 } 1 x=_{d f}[\lambda y \cdot E ! y \vee \neg E ! y] x
$$

Now we can easily derive:

$$
\begin{array}{ll}
\text { TB4 } & \neg \text { B1 } \\
& \text { Universal property is not conditioned. } \\
\text { TB5 } & I N \rightarrow(F x \wedge A ! x \wedge \neg G \text { xcon } G \rightarrow \neg A d F) \\
& \begin{array}{l}
\text { A property exemplified by an abstract object that is not a characteristic } \\
\text { coder of any property, is not an adherence. }
\end{array} \\
\text { TB6a } & \neg A d A ! \quad 6 \mathrm{~b} \neg A d 1 \\
& \begin{array}{l}
\text { Property of being an abstract and the universal property are not adher- } \\
\text { ences. }
\end{array}
\end{array}
$$
TB5 $I N \rightarrow(F x \wedge A ! x \wedge \neg \exists G x \operatorname{con} G \rightarrow \neg A d F)$
(AB0, D1, AB4) A property exemplified by an abstract object that is not a characteristic coder of any property, is not an adherence.
TB6a $\neg A d A$ !
$6 \mathrm{~b} \neg A d 1$
(AB4, D1, D4, D8)
Property of being an abstract and the universal property are not adher- ences.

On the basis of $\mathrm{AOT}_{A d B \mathfrak{I n}}[\mathfrak{B}]$ we may put a question related to the conjecture that the Bolzanian theory of ideas is inconsistent: What about the self-referentiality of adherences and the very concept of Inbegriff? The problem of whether some of Bolzano's considerations on ideas in themselves drift towards contradictions in the 
style of Russell's antinomy, was raised by Berg (1987) and Morscher (2007). ${ }^{12}$ The following passage recorded by Berg demonstrates that Bolzano was fully aware of the danger of an uncontrolled use of the concept of set:

Problems involved in the first concepts of the Theory of Quantities. The total of all $A$, where $A$ denotes an idea of limited scope, e.g. Man-is considered rather frequently. But as soon as we substitute for $A$ the widest of all ideas, namely, the idea of Anything, then the problem arises that the idea: the total of all thing — or the total of all or the absolute All actually should comprehend even itself, since this All is something also; which is absurd, however (citation following Berg 1987, from Misscellanea mathematica, manuscript Dep. of the Austrian National Library, Series nova 3455, 1968)

Berg claims that these explanations should be linked with multitudes which are sets in sense of Mengen and not with Inbegriffen of objects that stands under (fallen unter) ideas in themselves. Actually, Bolzano considered the idea of an object in general (and the idea of something) that stands under itself (WL, I.459, WL I 496). Had he also considered the idea of all non-self-referential ideas (which do not stand under themselves), then perhaps he would have become aware of the dangers of antinomial Russellian's reasoning. Indeed, if we assume some kind of comprehension schema for multitudes of objects standing under ideas (cf. WL, I.468-473), then we have the following problem: if, on the one hand, the idea of all ideas which do not stand under themselves is self-referential (means: stands under itself), then it is non-self-referential; on the other hand: if it is non-self-referential (i.e. it does not stand under itself), then it stands under the idea of all selfreferential ideas, hence it is self-referential. We leave the open question whether Bolzano's system was explicit enough to allow the reconstruction of such antinomy [and so to justify the formal approaches of Berg (1987) and Morscher (2007)].

In frame of our proposal, it is worth noticing that AOT already allows to speak in a consistent way about the (non)self-referentiality of some abstract individuals in the sense of the predicate $\varepsilon$.

Let us introduce the following definition:

D9 $\operatorname{ref}(x)={ }_{d f} x \varepsilon x$ (self-referentiality of individuals)

From D1, A2, D6, and D9 we get:

T5 $\operatorname{ref}(x) \rightarrow A ! x$

Only abstract objects may be self-referential.

We define the individual characterizing a universal property as follows:

$\mathrm{D} 10 \quad x=\mathrm{u}={ }_{d f} x \operatorname{con} 1 \quad$ (universal abstract)

The universal abstract is self-referential and the invisible abstract is non-selfreferential:
T6a $\operatorname{ref}(\mathrm{u})$
$6 \mathrm{~b} \neg \operatorname{ref}(\mathrm{o})$
(D10, D4,T4b)

\footnotetext{
12 Professor Morscher also investigated other inconsistency problems in Bolzano's views. For instance, he reconstructed a form of the liar paradox and the paradox of non-self-referential properties from Bolzano's texts.
} 
An attempt to test the resistance of $\mathfrak{I} \mathfrak{n}$ to Russell's antinomy, would not succeed in a way similar to that of Clark, ${ }^{13}$ although the consequent of our AB0 breaks the restriction put on Comp $\lambda$. Let us assume $I N$ and the definition $\mathfrak{I} \mathfrak{n}^{-} x={ }_{d f} \neg \mathfrak{I} \mathfrak{n} x$. We call $\mathfrak{i}^{-}$the characteristic coder of $\mathfrak{\Im} \mathfrak{n}^{-}$. From $\mathfrak{I} \mathfrak{n} \mathfrak{i}^{-}$we get only $A d \mathfrak{I} \mathfrak{n}^{-}$and $\neg \mathfrak{I} \mathfrak{n} \mathfrak{i}^{-}$ with $\mathfrak{i}^{-}$con $\mathfrak{\Im} \mathfrak{n}^{-}$imply only $\neg A d \mathfrak{I} \mathfrak{n}^{-}$.

The connection between the self-referentiality of the characteristic coder of $\mathfrak{I} \mathfrak{n}$ and the fact that $\mathfrak{I} \mathfrak{n}$ is an adherence is described by the following theorem of $\mathrm{AOT}_{A d B \mathfrak{I n}}[\mathfrak{B}]$ :

TB7 IN $\rightarrow(x \operatorname{con} \mathfrak{I} \mathfrak{n} \rightarrow(\operatorname{ref}(x) \leftrightarrow A d \mathfrak{I}))$

1. $I N$

ass

2. $x \operatorname{con} \mathfrak{I n}$

ass

3a. $x \varepsilon x$

ass

4. $\exists F(F x \wedge x \neq \circ \wedge x \operatorname{con} F)$

D6, 3a

5. $\mathfrak{I n} x$

$2,4, \mathrm{~T} 3 \mathrm{a}$

6. $\exists F(A d F \wedge x \operatorname{con} F)$

$1,5, \mathrm{AB} 0$

7. $A d \mathfrak{I n}$

$6,2, \mathrm{~T} 3 \mathrm{a}$

8b. $\neg \mathfrak{I n} x$

ass

9. $\neg A d \mathfrak{I n}$

$2,8 \mathrm{~b}$

10. $I N \rightarrow(x \operatorname{con} \mathfrak{I n} \rightarrow(\operatorname{ref}(x) \leftrightarrow \operatorname{Ad} \mathfrak{I n}))$

D9, 1, 2, 3a, 7, 8b, 9 .

In fact, the possibility to speak consistently about Inbegriff in the sense of $\mathfrak{I n}$ depends on non-self-referentiality of adherences.

Let us take the following definition of (weak) self-reference of properties:

$$
\mathrm{D} 11 \operatorname{RefF}=_{d f} \exists x(x F \wedge F x)
$$

The self-referentiality of properties consists in the fact that some individuals representing them belong also to their extensions. We can easily notice that

$$
\text { TB8 } \forall F(F \subset S \rightarrow \neg \operatorname{Ref} F) \text {, where } F \subset G=_{d f} \forall x(F x \rightarrow G x)(\mathrm{D} 11, \mathrm{D} 2)
$$

We cannot transfer the above connection to intensional subsumption.

Now, the idea of taking adherences to be non-self-referential properties in the sense of $\neg$ Ref leads to Russell's antinomy on the ground of $\mathrm{AOT}_{\operatorname{AdB\mathfrak {I}}}[\mathfrak{B}]$.

\footnotetext{
13 The purpose of Clark's argument is to demonstrate that we fall into Russell's antinomy when $\operatorname{Comp} \lambda$ is used without the restriction to propositional formulas. Let as assume that we accept the definition $R={ }_{d f} \lambda x . \forall F(x F \rightarrow F x)$. The complement of $R$ is described in the following way: $R^{-}=_{d f} \lambda x \cdot \exists F(x F \wedge \neg F x)$. We take $\mathfrak{a}$ such that: $\mathfrak{a} c o n R^{-}$. The question now is whether $R^{-} \mathfrak{a}$ or $\neg R^{-} \mathfrak{a}$ ? We assume that $R^{-} \mathfrak{a}$. We get $\exists F(\mathfrak{a} F \wedge \neg F \mathfrak{a})$. We know that $\mathfrak{a} R^{-} \wedge \neg R^{-} \mathfrak{a}$. So: $\neg R^{-} \mathfrak{a}$. Now we assume that $\neg R^{-} \mathfrak{a}$. Then $R \mathfrak{a}$, and from this $\forall F(\mathfrak{a} F \rightarrow F \mathfrak{a})$. By logic we get: $\mathfrak{a} R^{-} \rightarrow R^{-} \mathfrak{a}$, so: $R^{-} \mathfrak{a}$. This chain of reasoning refers to explanations given in Rapaport (1978).
} 
We define an empty property as follows:

$\mathrm{D} 12 \quad 0 x=_{d f}[\lambda y \cdot E ! y \wedge \neg E ! y] x$

Extensional identity of properties is understood as usual:

D13 $F \equiv G=_{d f} \forall x(F x \leftrightarrow G x)$

The following equivalence enables to reach contradiction in Russel's style:

Add $A d F \leftrightarrow \neg \operatorname{RefF} \wedge F \not \equiv 0$

It is assumed that $I N$

Let $\mathfrak{i}$ be the characteristic coder of $\mathfrak{I n}: \quad \mathfrak{i}$ con $\mathfrak{I n}$

$(2: \mathrm{T} 4 \mathrm{~b}, 1)$

It is also a coder of $\mathfrak{I n}: \quad \mathfrak{i} \mathfrak{I n}$

Now we assume that $\mathfrak{i} \varepsilon \mathfrak{i}$

We get $A d \mathfrak{I n}$

From Add, D11 we obtain $\neg \mathfrak{I n i}$

Now we take TB1, 2, 6 and we have $\neg(\mathfrak{i} \varepsilon \mathfrak{i})$

We assume that $\neg(\mathfrak{i} \varepsilon \mathfrak{i})$

From 1, 2, and 8 with TB7: $\neg A d \mathfrak{I n}$.

But also $\mathfrak{I n} \not \equiv 0$ because of TB2 and Add.

We take again Add and using D11 we obtain $\exists x(x \mathfrak{I n} \wedge \mathfrak{I n} x)$

Let us take $a$ for $x$ : $a \mathfrak{I n} \wedge \mathfrak{I n} a$

From $10 \mathrm{a}, \mathrm{AB} 0$ and 1 we get $\exists F(A d F \wedge a c o n F)$

We take $F^{\prime}$ for $F$ : $A d F^{\prime} \wedge a c o n F^{\prime}$

From 10a. we have $a \mathfrak{I n}$, so with $11 \mathrm{a}$ and T3b: $\quad F^{\prime}=\mathfrak{I n}$

Next, we obtain acon $\mathfrak{I n}$

By D6, 10a, and 9: aعa

But we have $10 \mathrm{a}$, and 2 , and 13 , again by $\mathrm{A} 4$ : $\mathfrak{i} \varepsilon \mathfrak{i}$

From $4-7$ and $8-15$ we get $\mathfrak{i} \varepsilon \mathfrak{i} \leftrightarrow \mathfrak{i} \not \mathfrak{i}$.

$\mathrm{AOT}_{A d B \mathfrak{n} \mathfrak{n}}[\mathfrak{B}$, Add $]$ is inconsistent. Assuming only right-sided implication from Add does not lead to contradiction (cf. our Aczel structure $\mathcal{A}_{\mathbf{I}}^{\mathcal{B}}$ in (4.2)).

One thing to notice is that in our formalization we can consistently speak of the self-referentiality of the characteristic coder of $\mathfrak{I} \mathfrak{n}$ (if $I N$ is fulfilled). It will be shown, that in the case of Aczel's semantics, there are models for $\operatorname{AOT}_{A d B \mathfrak{I n}}[\mathfrak{B}, I N]$ that allow its self-referentiality. 


\section{Two Interpretations of $\mathrm{AOT}_{A d B \mathfrak{I} \mathfrak{}}[\mathfrak{B}]$}

We consider two semantic frameworks originally designed for different versions of Zalta's formalism. The first one was suggested to Zalta by D. Scott. Classical firstorder fragment of the theory of abstracts objects is sound in this semantics (Zalta 1983). Our AOT AdBIn $_{n}$ is a system of this kind. The second approach follows ideas that Zalta owes to P. Aczel. This interpretation was developed for certain S5 extension of Zalta's system (Zalta 1997, 1999). Here we will simplify Aczel's semantics and adopt it to our theory as more 'attractive' than the first one.

Both interpretations taken in account, are based on the same idea of expressing extensional and intensional nature of predicates by the simultaneous use of two functions. Extension function assigns to every property corresponding to some predicate, a set of individuals that posses this property. Intension function assigns to every property a set of individuals which represent it. In the universe of individuals we meet concrete (ordinary) and abstract individuals. Objects of the first kind do not belong to a value of any intension function but individuals of both kinds can belong to extensions of properties. The way of treating abstracts as elements of extensions is different in the considered approaches. In Aczel's semantics there is used a technical trick consisting of an imitation of abstracts by their proxies. Thanks to this we can consider models including self-referential properties in the sense of D11 and such that do not have all abstracts in their extensions.

Scott and Aczel structures presented here are adequate to the fragment of Zalta's theory used in our formalization. However, they verify different sets of formulas and so they can behave in different ways when they are used for interpretation of some $\mathrm{AOT}_{A d B \Im \mathfrak{n}}$ extensions. Indeed, as we will see, Scott's semantics does not allow to consider structures with non-empty set of adherences. This problem is not present in Aczel structures.

\subsection{Scott's Semantics for $\mathrm{AOT}_{A d B \mathfrak{N} \mathfrak{n}}[\mathfrak{B}]$}

Following Zalta (1983), we formulate a definition of a Scott structure:

DF1 A Scott structure is a 4-tuple $S=<\mathcal{E}$, Prop, ext, int $>$, such that:

(i) $\mathcal{E}$ is the set of concrete individuals, possibly empty

(ii) Prop, is a set of properties ${ }^{s 14}$ defined as a Cartesian product of the set containing two constants $\{+,-\}$ and the set $2^{\mathcal{E}}$ : Prop $=\{+,-\} \times 2^{\mathcal{E}}$.

For elements of Prop $\langle+, X\rangle,\langle-, X\rangle$ we take notation $+X,-X$ and we represent them using variables $p, p^{\prime}, \ldots(p=+X$ is an abbreviation for: $p=+X$ or $p=-X)$.

Elements of $2^{\text {Prop }}$ are called abstract individuals $^{s}$ and the sum: $\mathcal{E} \cup 2^{\text {Prop }}$ is the set of all individuals-objects ${ }^{s}$. We use $o, o^{\prime}$ to represent elements of $\mathcal{E} \cup 2^{\text {Prop }}$.

\footnotetext{
${ }^{14}$ We use superscripts: $s$ and $a$ to remember the difference between approaches inspired by Scott and Aczel.
} 
(iii) ext : Prop $\rightarrow 2^{\mathcal{E} \cup 2^{\text {Prop }}}$ is the function of extension ${ }^{s}$ of properties, which fullfills the following condition:

$o \in \operatorname{ext}(p) \Longleftrightarrow \exists_{X \in 2^{\varepsilon}}(p= \pm X$ and $o \in X)$ or $\exists_{X \in 2^{\varepsilon}}\left(o \in 2^{\text {Prop }}\right.$ and $\left.p=+X\right)$

(iv) int : Prop $\rightarrow 2^{\mathcal{E} \cup 2^{\text {Prop }}}$ is the function of intension ${ }^{s}$ of properties, which fullfills the following condition:

$o \in \operatorname{int}(p) \Longleftrightarrow o \in 2^{\text {Prop }}$ and $p \in o$.

We connect Scott structures with AOT language.

DF2 For any given $S=<\mathcal{E}$, Prop, ext, int $>$, valuation $v$ is a function such that:

(i) $v(x) \in \mathcal{E} \cup 2^{\text {Prop }}$, (ii) $v(\tau) \in$ Prop and $v(E$ !) $=-\mathcal{E}$

(iii) $v(\tau x)=1 \Longleftrightarrow v(x) \in \operatorname{ext}(v(\tau))$, otherwise: $v(\tau x)=0$

(iv) $v(x \tau)=1 \Longleftrightarrow v(x) \in \operatorname{int}(v(\tau))$, otherwise: $v(x \tau)=0$

(v) $v\left(x={ }_{E} y\right)=1 \Longleftrightarrow v(x)=v(y)$ and $v(x) \in-\mathcal{E}$, otherwise: $v\left(x={ }_{E} y\right)=0$

(vi) $v([\lambda x . \varphi x]) \in \operatorname{Prop} \operatorname{ext}(v([\lambda x . \varphi x]))=\left\{o: v^{\prime}(x)=o\right.$ and $v^{\prime}(\varphi x)=1$, for every $v^{\prime}$ which is $x$-variant of $\left.v\right\} \operatorname{int}(v([\lambda x . \varphi x])$ like in DF1, (iv).

(vii) Conditions for: $\neg, \rightarrow, \forall$ are classical

DF3 A formula $\varphi$ is valid in $S$ iff for every valuation $v$ in $S: v(\varphi)=1$.

If $\varphi$ is AOT derivable, then $\varphi$ is valid in every $S$ (based on Zalta 1983, 161-164).

Let us mention that Scott's semantics is extensional in this sense that the formula:

Ext $F \equiv G \rightarrow F=G$

is valid in every $S$ (cf. Zalta 1983, 160-161). The implication converse to Ext follows directly from D2, A4, D13.

Now we consider an interpretation of the $\mathrm{AOT}_{A d B}$ language and take a 6-tuple $\mathcal{S}^{\mathcal{B}}=\left\langle\mathcal{E}\right.$, Prop, ext, int $\left., A d^{*}, B^{*}\right\rangle$ such that $S=\langle\mathcal{E}$, Prop, ext, int $\rangle$ is a Scott structure and $A d^{*}, B^{*} \subseteq$ Prop. We assume also that: $p \in B^{*} \Longrightarrow$ $\left\{\operatorname{ext}(p) \subseteq \mathcal{E}\right.$ or $\exists_{o}\left[o \in \operatorname{ext}(p)\right.$ and $\exists_{o^{\prime}}\left(o^{\prime} \notin \operatorname{ext}(p)\right.$ and $\exists_{p^{\prime}}\left(o^{\prime} \in \operatorname{ext}\left(p^{\prime}\right)\right.$ and $o^{\prime} \neq 0$ and $\left.\left.\forall_{p^{\prime \prime}}\left(o \in \operatorname{int}\left(p^{\prime \prime}\right) \Leftrightarrow\left(\operatorname{int}\left(p^{\prime \prime}\right)=\operatorname{int}\left(p^{\prime}\right)\right)\right)\right]\right\}$.

We extend DF2 by the following conditions:

(ii') $v(S)=v(E !)$

(iii') $v(A d \tau)=1 \Longleftrightarrow v(\tau) \in A d^{*}$, otherwise: $v(A d \tau)=0$

(iii”) $v(B \tau)=1 \Longleftrightarrow v(\tau) \in B^{*}$, otherwise: $v(B \tau)=0$

All formulas which are valid in $\mathcal{S}$ are also valid in any $\mathcal{S}^{\mathcal{B}}$.

All $\mathrm{AOT}_{A d B \mathfrak{I n}}$ derivable formulas are valid in every $S^{\mathcal{B}}$. 
Let us take the structure $\boldsymbol{S}^{\boldsymbol{B}}$ with $\mathcal{E}=\{s\}$, Prop $=\{+\{s\},-\{s\},+\emptyset,-\emptyset\}$, functions $\boldsymbol{e x t}$ and $\boldsymbol{i n t}$ are determined as in DF1, and $\boldsymbol{A d}^{*}=\emptyset$.

We can observe that:

\section{Th1 AOT $_{A d B \Im \mathfrak{m}}[\mathfrak{B}]$ is valid in $\mathcal{S}^{\boldsymbol{B}}$}

We note that $I N$ is $\mathcal{S}^{\mathcal{B}}$ valid for property $-\emptyset$. AB0 is valid for: $v(\mathfrak{I n})=-\emptyset$. From DF1 we know that $\operatorname{ext}(\mathfrak{I n})=\emptyset, \boldsymbol{i n t}(\mathfrak{I n})=\left\{o \in 2^{\text {Prop }}:-\emptyset \in o\right\}$. AB1 is $\boldsymbol{S}^{\boldsymbol{B}}$ valid because $\left.v(S) \neq \emptyset(v(S)=-\{s\}), \boldsymbol{e x t}(v(S))=\{s\}, \operatorname{int}(v(S))=\left\{o \in 2^{\text {Prop }}:-\{s\} \in o\right\}\right)$. AB2 is $\boldsymbol{S}^{\boldsymbol{B}}$ valid because $v(\exists x \mathfrak{I} \mathfrak{n} x)=0$; AB4 is $\boldsymbol{S}^{\boldsymbol{B}}$ valid because $v(\exists F A d F)=0$.

The above theorem is actually mentioned by Świętorzecka (2017) and it shows the consistency of $\mathrm{AOT}_{A d B \mathfrak{I n}}[\mathfrak{B}]$. However, it refers to rather 'trivial' structures in which there are no adherences (sic!). The search of more interesting Scott structures is rather doomed to failure in view of the following:

Th2 $\exists x(A ! x \wedge F x) \rightarrow \forall x(A ! x \rightarrow F x) \quad$ is valid in every $S$

Let us remind the condition ext: $o \in \operatorname{ext}(p) \Longleftrightarrow \exists_{X \in 2^{\varepsilon}}(p=+X$ and $o \in X)$ or $\exists_{X \in 2^{\varepsilon}}\left(o \in 2^{\text {Prop }}\right.$ and $\left.p=+X\right)$. We take any $p^{*}$ and some $o^{*}$ such that they validate $\exists x(A ! x \wedge F x)$. This means that $o^{*} \in 2^{\text {Prop }}$ and $o^{*} \in \operatorname{ext}\left(p^{*}\right)$. If $o^{*} \in 2^{\text {Prop }}$ then $o^{*} \notin \mathcal{E}$ and so we know that $\exists_{X \in 2^{\varepsilon}}\left(p^{*}=+X\right)$. But from the condition for ext by classical logic we have: $\exists_{X \in 2^{\varepsilon}}(p=+X) \wedge o \in 2^{\text {Prop }} \rightarrow o \in \operatorname{ext}(p)$, for any $o$.

Theorem Th2 expresses the idea of reloading abstracts in extensional contexts.

Now we can observe that

Th3 IN is not valid in any $\mathcal{S}^{\mathcal{B}}$ structure with $A d^{*} \neq \emptyset$.

Indirect proof. We assume that $\boldsymbol{Z} x \leftrightarrow \exists F(A d F \wedge x \operatorname{con} F)$ is valid in a given $\boldsymbol{S}^{\boldsymbol{B}}(1)$. Because $\boldsymbol{A d}^{*} \neq \emptyset$, T4b and D5 for certain $x$ it is $\boldsymbol{S}^{\boldsymbol{B}}$ valid: $\exists_{x}(\boldsymbol{Z} x \wedge A ! x)$ (2). But from Th2 we get as $\boldsymbol{S}^{\boldsymbol{B}}$ valid: $\forall x(A ! x \rightarrow \boldsymbol{Z} x)$ (3) and by T1, D4 also $\boldsymbol{Z}$ o is $\boldsymbol{S}^{\boldsymbol{B}}$ valid (4). However, from T1, D4 we know that $\neg \exists F\left(\right.$ ocon $F$ ) is AOT $_{A d B \Im \mathfrak{n}}$ thesis (5) so: $\neg Z$ o is $\boldsymbol{S}^{\boldsymbol{B}}$ valid (6) which contradicts (4).

In this way we know that:

Th4 There is no $\mathcal{S}^{\mathcal{B}}$ structure with $A d^{*} \neq \emptyset$ verifying $\mathrm{AOT}_{A d B \Im \mathfrak{n}}[\mathfrak{B}, I N]$

This is the reason why we look for another semantics.

\subsection{Aczel's Semantics and Its Application to $\mathrm{AOT}_{A d B \Im \mathfrak{m}}[\mathfrak{B}]$}

In view of Th2 it is obvious that adding to the theory AOT interpreted in Scott structures an assumption about existence of any property which has in its extension some but not all abstract objects results in contradiction. Aczel's semantics is not so 'bearish' and it falsifies the formula from Th2. In this frame we can speak about self-referential properties which do not contain in their extensions all abstracts. This effect is due to adding to the set of ordinary objects, mentioned proxies of abstracts that 
are 'invisible' on the level of an object language. Another intensional profit coming from Aczel's semantics is the possibility to distinguish between identity of extensions and identity of intensions of properties. The formula Ext is not valid here. This effect is irrelevant to our particular approach although one could think about this advantage in connection with Bolzano's views about the difference between extensions and intensions of ideas in themselves (WL, I.428-I.459).

Let us define Aczel structure in the following way:

DF4 An Aczel structure is a 7-tuple $\mathcal{A}=\langle\mathcal{E}$, Ps, Prop, e, | |, ext, int $\rangle$, such that:

(i) $\mathcal{E}$ is, as in DF1, the set of concrete individuals, possibly empty ${ }^{15}$

(ii) Ps is a nonempty set of objects thought as proxies of abstract objects ${ }^{a}$ introduced in (iv); Ps $\neq \emptyset$ and Ps $\cap \mathcal{E}=\emptyset$

(iii) Prop is a set of properties ${ }^{a}: \mathrm{p}, \mathrm{p}^{\prime}, \ldots$, such that $e \in$ Prop, $\operatorname{card}\left(\right.$ Prop) $\geq \operatorname{card}\left(2^{\mathcal{E} \cup \mathrm{Ps}}\right)$

(iv) Elements of $2^{\text {Prop }}$ are abstracts $^{a}$ (imitated by individuals from Ps)

Elements of $\mathcal{E} \cup \mathrm{Ps}$ are called ordinary* objects. The sum of ordinary* and abstract objects $^{a}: \mathcal{E} \cup \operatorname{Ps} \cup 2^{\text {Prop }}$ is the domain of all objects ${ }^{a}$ (represented by variables $o, o^{\prime}$ ).

(v) ||$: \mathcal{E} \cup 2^{\text {Prop }} \rightarrow \mathcal{E} \cup \mathrm{Ps}$ is an imitation function $o \in \mathcal{E} \Longrightarrow|o|=o ; o \in 2^{\text {Prop }} \Longrightarrow \exists_{o^{\prime}}\left(o^{\prime} \in \operatorname{Ps}\right.$ and $\left.|o|=o^{\prime}\right)$

(vi) ext : Prop $\rightarrow 2^{\mathcal{E} \cup \mathrm{Ps}}$ is the function of extension ${ }^{a}$ which assigns to every property $^{a}$ its extension and ext $(e)=\mathcal{E}$

(vii) int : Prop $\rightarrow 2^{\mathcal{E} \cup 2^{\text {Prop }}}$ is the function of intension ${ }^{a}: o \in \operatorname{int}(\mathrm{p}) \Longleftrightarrow o \in 2^{\text {Prop }}$ and $\mathrm{p} \in \boldsymbol{o}$.

DF5 For any given Aczel structure $\mathcal{A}$ we define a valuation v in $\mathcal{A}$ :

(i) $\mathrm{v}(x) \in \mathcal{E} \cup 2^{\text {Prop }}$, (ii) $\mathrm{v}(\tau) \in \operatorname{Prop}$, and $\mathrm{v}(E$ ! $)=\mathrm{e}$

(iii) $\mathrm{v}(\tau x)=1 \Longleftrightarrow|\mathrm{v}(x)| \in \operatorname{ext}(\mathrm{v}(\tau))$, otherwise: $\mathrm{v}(\tau x)=0$

(iv) $\mathrm{v}(x \tau)=1 \Longleftrightarrow \mathrm{v}(x) \in \operatorname{int}(\mathrm{v}(\tau))$, otherwise: $\mathrm{v}(x \tau)=0$

(v) $\mathrm{v}\left(x={ }_{E} y\right)=1 \Longleftrightarrow|\mathrm{v}(x)|=|\mathrm{v}(y)|$ and $\mathrm{v}(x) \in \mathcal{E}$, otherwise: $\mathrm{v}\left(x={ }_{E} y\right)=0$

(vi) $\operatorname{ext}(v([\lambda x . \varphi x]))=\left\{o:\left|\mathrm{v}^{\prime}(x)\right|=o\right.$ and $\mathrm{v}^{\prime}(\varphi x)=1$, for every $\mathrm{v}^{\prime}$ which is $x$-variant of $\mathrm{v}\} \operatorname{int}(v([\lambda x . \varphi x])$ like in DF4, (vii).

(vii) Operators $\neg, \rightarrow, \forall$ have classical meaning.

We define validity as usual:

DF6 $\varphi$ is valid in $\mathcal{A}$ iff for every $\mathcal{A}$ valuation v: $\mathrm{v}(\varphi)=1$.

\footnotetext{
15 There is assumed that $\mathcal{E} \neq \emptyset$ in the modal version of Aczel's semantics (cf. Zalta 1997, 271). We do not need to take here such an assumption.
} 
Zalta sketches a proof of soundness of his S5 extension of the theory of abstract objects in a richer version of the above semantics (cf. Zalta 1997, 276-278). Thus we can say that if $\varphi$ is AOT derivable, then $\varphi$ is valid in every structure $\mathcal{A}$.

Now we proceed in a similar way as in 4.1.

We take $\mathcal{A}^{\mathcal{B}}=\left\langle\mathcal{E}\right.$, Ps, Prop, e, | |, ext, int, $\left.A d^{*}, B^{*}\right\rangle$, where

$\langle\mathcal{E}$, Ps, Prop, e, | |, ext, int $\rangle$ is $\mathcal{A}$ structure and $A d^{*}, B^{*} \subseteq$ Prop. We assume that: $p \in B^{*} \Longrightarrow\left\{\operatorname{ext}(\mathrm{p}) \subseteq \mathcal{E}\right.$ or $\exists_{o}\left[o \in \operatorname{ext}(\mathrm{p})\right.$ and $\exists_{o^{\prime}}\left(o^{\prime} \notin \operatorname{ext}(\mathrm{p})\right.$ and $\exists_{\mathrm{p}^{\prime}}\left(o^{\prime} \in \operatorname{ext}\left(\mathrm{p}^{\prime}\right)\right.$ and $o^{\prime} \neq o$ and $\left.\left.\forall_{\mathrm{p}^{\prime \prime}}\left(o \in \operatorname{int}\left(\mathrm{p}^{\prime \prime}\right) \Leftrightarrow\left(\operatorname{int}\left(\mathrm{p}^{\prime \prime}\right)=\operatorname{int}\left(\mathrm{p}^{\prime}\right)\right)\right)\right]\right\}$.

We extend $\mathcal{A}$ valuation $\mathrm{v}$ by conditions expressed in the same way as (ii'), (iii'), (iii") for $S^{\mathcal{B}}$ valuation (DF2).

We notice that

Th5 $\mathrm{AOT}_{A d B \mathfrak{I n}}[\mathfrak{B}]$ is valid in every $\mathcal{A}^{\mathcal{B}}$ with $\mathcal{E} \neq \emptyset$ and $A d^{*}=\emptyset$.

We prove it in two steps. 1. If $\exists_{\mathrm{p}, \mathrm{p}^{\prime} \in \operatorname{Prop}}\left(\mathrm{p} \neq \mathrm{p}^{\prime}\right.$ and $\left.\operatorname{ext}(\mathrm{p})=\operatorname{ext}\left(\mathrm{p}^{\prime}\right)=\emptyset\right)$ then $\mathrm{AB} 0, \mathrm{AB} 2, \mathrm{AB} 4$ are valid because $I N$ is not valid in $\mathcal{A}^{\mathcal{B}}$. $\mathrm{AB} 1$ is valid because $\exists x S x$ is valid. 2. $\exists_{\mathrm{p} \in \operatorname{Prop}}^{1}(\operatorname{ext}(\mathrm{p})=\emptyset)$ called: $\mathrm{p}^{*}$. We take $\mathrm{v}(\mathfrak{I n})=\mathrm{p}^{*}$. AB0 and AB4 are

valid because $\exists F A d F$ is not valid. AB1 is valid because $\exists x S x$ is valid, AB2 is valid because $\exists x \mathfrak{I} \mathfrak{n} x$ is not valid in $\mathcal{A}^{\mathcal{B}}$.

It is important to recall that Aczel structures are intensional in this sense that Ext is not valid in every $\mathcal{A}$. ${ }^{16}$ Of course, when in $\mathcal{A}$ there are two properties with the same extension, they cannot be named by the same term. This is just the case considered in part 1 of the proof of Th5. Thus, in order to speak about a possible extension of $\mathrm{AOT}_{A d B \Im \mathfrak{n}}[\mathfrak{B}]$ by $I N$, we take into account $\mathcal{A}^{\mathcal{B}}$ structures that are extensional at least in respect to the property that has as its extension the set of all adherential ideas (ie. characteristic coders of adherences—cf. D5):

$$
\text { (IN) } \begin{aligned}
& \forall_{\mathrm{p}, \mathrm{p}^{\prime} \in \operatorname{Prop}}\left(\operatorname{ext}(\mathrm{p})=\operatorname{ext}\left(\mathrm{p}^{\prime}\right)=\left\{o: \forall_{\mathrm{p}^{\prime \prime} \in \operatorname{Prop}}\left(\mathrm{p}^{\prime \prime} \in A d^{*} \Longleftrightarrow\left|\left\{\mathrm{p}^{\prime \prime}\right\}\right|=o\right\}\right.\right. \\
\left.\Longrightarrow \mathrm{p}=\mathrm{p}^{\prime}\right) &
\end{aligned}
$$

We call $\mathcal{A}_{\text {IN }}^{\mathcal{B}}$ structure $\mathcal{A}^{\mathcal{B}}$ which fullfils IN.

When we consider $I N$ as a desirable extension of our theory, we should recall the problematic formula on reloading abstracts from Th2. In case of $\mathcal{A}$ structures we see that:

Th6 $\forall F(\exists x(A ! x \wedge F x) \rightarrow \forall x(A ! x \rightarrow F x))$ is valid in every $\mathcal{A}$ structure with $\operatorname{card}(\mathrm{Ps})=1$.

Indirect proof. We assume that there is $\mathcal{A}$ structure with $\operatorname{card}(\mathrm{Ps})=1$ in which $\exists x\left(A ! x \wedge F^{*} x\right)$ is valid (1) and $\exists x\left(A ! x \wedge \neg F^{*} x\right)$ is valid (2). We consider certain $\mathrm{v}$ valuation such that $\mathrm{v}\left(F^{*}\right) \in$ Prop and its two variants $\mathrm{v}^{\prime}$ and $\mathrm{v}^{\prime \prime}:\left|\mathrm{v}^{\prime}(x)\right| \notin \mathcal{E}$ and $\left|\mathrm{v}^{\prime}(x)\right| \in \operatorname{ext}\left(\mathrm{v}\left(F^{*}\right)\right)(3)$ (because of (1), DF5), $\left|\mathrm{v}^{\prime \prime}(x)\right| \notin \mathcal{E}$ and $\left|\mathrm{v}^{\prime \prime}(x)\right| \notin \operatorname{ext}\left(\mathrm{v}\left(F^{*}\right)\right.$ ) (4) (because of (2), DF5). Let us name individuals assigned by $\mathrm{v}^{\prime}$ and $\mathrm{v}^{\prime \prime}$ to $x$ :

${ }^{16}$ All $\mathcal{A}$ structures with $\operatorname{card}(\operatorname{Prop})>\operatorname{card}\left(2^{\mathcal{E} \cup \mathrm{Ps}}\right)$ are of this kind. 
$\mathrm{v}^{\prime}(x)=o$ and $\mathrm{v}(\mathrm{x})^{\prime \prime}=o^{\prime}$. From the fact that $\left|\mathrm{v}^{\prime}(x)\right|,\left|\mathrm{v}^{\prime \prime}(x)\right| \notin \mathcal{E}(3,4)$ and from DF5 (i), DF4 (v) we know that $o, o^{\prime} \in 2^{\text {Prop }}(5)$. Then there is some $d \in \mathrm{Ps}:\left|o^{\prime}\right|=d$ and $d \in \operatorname{ext}\left(\mathrm{v}\left(F^{*}\right)\right)(6)$ and some $d^{\prime} \in \mathrm{Ps}:\left|o^{\prime}\right|=d^{\prime}$ and $d^{\prime} \notin \operatorname{ext}\left(\mathrm{v}\left(F^{*}\right)\right)(7)$. But $\operatorname{card}(\mathrm{Ps})=1$. So we get contradiction from (6) and (7).

Now we can say that

Th7 There is no $\mathcal{A}^{\mathcal{B}}$ structure with $A d^{*} \neq \emptyset$ and $\operatorname{card}(\mathrm{Ps})=1$ verifying $\mathrm{AOT}_{A d B \mathfrak{N} \mathfrak{n}}[\mathfrak{B}, I N]$

Indirect proof. If $\mathcal{A}^{\mathcal{B}}$ fulfills IN, then we use Th6, otherwise we get contradiction by logic.

Finally, we give an example of intensional $\mathcal{A}_{\text {TN }}^{\mathcal{B}}$ structure with $\operatorname{card}(\mathrm{Ps})>1$ and $A d^{*} \neq \emptyset$, which may be used as an interpretation of $\mathrm{AOT}_{A d B \mathfrak{I n}}[\mathfrak{B}, I N]$.

We consider $\mathcal{A}_{\text {TN }}^{\mathcal{B}}$ such that:

(i) $\mathcal{E}=\{s\}$; (ii) $\operatorname{Ps}=\{i, d\}$; (iii) Prop $=\left\{\mathrm{e}, \bigvee, \bigwedge\right.$, In, $\left.\mathrm{D}, \mathrm{eIn}, \mathrm{eD}, \operatorname{InD}, \mathrm{e}^{*}\right\}$; (iv) abstracts $^{a}:\{X: X \subseteq$ Prop $\} ;$ (v) e is thought as an adherence, such that $|\{\mathrm{e}\}|=i$. Next conditions for the imitation function are: $\forall_{o \in 2^{\text {Prop }}}(\operatorname{card}(o)>1 \Longrightarrow|o|=d)$; $|\{\operatorname{InD}\}|=|\{\bigvee\}|=|\{\mathrm{D}\}|=|\{\mathrm{eD}\}|=|\{\operatorname{InD}\}|=d, \quad$ for $\quad$ remaning abstracts: $\bigwedge$, In, $e$ In, $e^{*}$ the function $\|$ is not determined; (vi) we decide that: $\operatorname{ext}(\mathrm{e})=\operatorname{ext}\left(\mathrm{e}^{*}\right)=\{s\}, \operatorname{ext}(\bigvee)=\mathcal{E} \cup \operatorname{Ps}, \operatorname{ext}(\bigwedge)=\emptyset$, $\operatorname{ext}(\operatorname{In})=\{i\}, \operatorname{ext}(\mathrm{D})=\{d\}, \operatorname{ext}(\mathrm{eIn})=\{s, i\}, \operatorname{ext}(\mathrm{eD})=\{s, d\}, \operatorname{ext}(\operatorname{InD})=\{i, d\} ; \quad(\mathrm{vii})$ int assigns to every property a set of abstracts ${ }^{a}$ as in DF4, (vii); (viii) in addition, we assume that $\forall_{\mathrm{p} \in \text { Prop }}\left(\mathrm{p} \in \boldsymbol{A} \boldsymbol{d}^{*} \Longleftrightarrow|\{\mathrm{p}\}|=i\right)$ and $\bigvee \notin \boldsymbol{B}^{*}$, In $\in \boldsymbol{B}^{*}$.

Of course, In is a correlate of $\mathfrak{I n}-$ it is our Inbegriff. If we refer our language to $\mathcal{A}_{\mathrm{IN}}^{\mathcal{B}}$ in a way described in DF5, we see that all axioms of the considered theory are valid as well as the condition $I N$.

$\mathcal{A}_{\text {IN }}^{\mathcal{B}}$ is an intensional structure, because $\operatorname{ext}(\mathrm{e})=\operatorname{ext}\left(\mathrm{e}^{*}\right)$ but $\operatorname{int}(\mathrm{e}) \neq \operatorname{int}\left(\mathrm{e}^{*}\right)$ $\left(e \neq e^{*}\right)$ and this falsifies Ext. Let us now put the question about self-referentiality of In. Imitation function for In is not determined. However, if $|\{\operatorname{In}\}|=i$, then In is self-referential and In $\in \boldsymbol{A d}^{*}$. The same applies to $|\{\bigwedge\}|,\left|\left\{\mathrm{e}^{*}\right\}\right|,|\{\mathrm{eIN}\}|$. By this reason we can have at least one adherence (e) and most five adherences. The structure with only one adherence e verifies right-sided implication Add.

Our example shows that Aczel's semantics gives a formal framework for speaking in a consistent way about adherences and their collection that ground the existence of substances in Bolzanian style. Another thing is to provide a pragmatic justification for the considered structure (and for others that diverge even more from intuition than $\mathcal{A}_{\text {IN }}^{\mathcal{B}}$ ). However, this issue is far beyond the intended analysis.

Acknowledgements I thank Professor W. Künne for the extensive and valuable lessons of Bolzano's philosophy, which he gave me in Dubrovnik in 2017. I also thank Professor E. Morscher for help and important suggestions on the selection of the right source texts. Many content-specific decisions owe their consultations.

Funding Research was carried out within the project Logic, Concepts, and Communication (LogiCCom, IP-2014-09-9378) financed by Croatian Science Foundation (HRZZ).

Open Access This article is distributed under the terms of the Creative Commons Attribution 4.0 International License (http://creativecommons.org/licenses/by/4.0/), which permits unrestricted use, distribution, 
and reproduction in any medium, provided you give appropriate credit to the original author(s) and the source, provide a link to the Creative Commons license, and indicate if changes were made.

\section{References}

Aristotle (1928) Categoriae [Cat]; Metaphysica [Met]. In: The works of Aristotle translated into English. Transl. under the editorship of W. D. Ross, The Clarendon Press, Oxford

Berg J (1987) Is Russell's antinomy derivable in Bolzano's logic? Philos Nat 24(4):406-413

Bolzano B (1827) Athanasia oder Gründe für die Unsterblichkeit der Seele, Sulzbach, J. E. v. Seidel; used here: 2nd improved and enlarged edition (no longer anonymous): Sulzbach: J. E. v. Seidel, 1838; reprint: Frankfurt/M.: Minerva, 1970

Bolzano B (1833-44) Briefwechsel mit Franz Exner 1833-1844. Morscher E (ed). In: BGA 3.4/1, Stuttgart - Bad Cannstatt

Bolzano B (1837a) Wissenschaftslehre. Versuch einer ausführlichen und grösstentheils neuen Darstellung der Logik mit steter Rücksicht auf deren bisherige Bearbeiter [WL], 4 vols. In: BGA, 1.11/1-14/3, trans. and Rusnock P, George R (eds). 2014. Oxford University Press, Publisher

Bolzano B (1837b) Verschiedenheit zwischen Leibnitzens und meinen Ansiehten. BGA 2B(18/2):37-48

Bolzano B (1851) Paradoxes of the infinite. In: Steele DA (ed) 1950 London: Yale University Press, Routledge and Kegan Paul, and New Haven

Künne W (1997) Propositions in Bolzano and Frege. Gazer Philosophische Studien 53:203-240

Künne W (2008) Versuche über Bolzano/Essays on Bolzano. Academia Verlag, Sankt Augustin

Künne W (2015) On having a property. Corrigenda in Bolzano's Wissenschaftslehre. Grazer Philos Stud 91(1(1)):365-408

Lapointe S (2011) Bolzano's theoretical philosophy. An introduction. Palgrave Macmillan, London

Morscher E (2007) "Hintertürln" für Paradoxien in Bolzanos Logik. In: Studien zur Logik Bernard Bolzano's, Beiträge zur Bolzano-Forschung, vol. 20. Salzburg 2007, 157-167

Rapaport WJ (1978) Meinongian theories and a Russellian paradox. Nous 12:153-180

Schnieder B (2002) Substanz und Adhärenz-Bolzanos Ontologie des Wirklichen. Academia Varlag, Sankt Augustin

Świętorzecka K (2014) An argument for the existence of God by Bolzano. A formalization with a distinction between Menge and Inbegriff. Bull Sect Log 43(3):155-172

Świętorzecka K (2017) Bolzano's argument for the existence of substances: a formalization with two types of predication. Acta Anal. https://doi.org/10.1007/s12136-017-0317-4

Zalta E (1983) Abstract objects: an introduction to axiomatic metaphysics. D. Reidel, Dordrecht

Zalta E (1997) The modal object calculus and its interpretation. In: De Rijke M (ed) Advances in intensional logic. Kluwer, Dordrecht, pp 249-279

Zalta E (1999) Natural numbers and natural cardinals as abstract objects: a partial reconstruction of Frege's Grundgesetze in object theory. J Philos Log 28(6):619-660 